\title{
Evaluation of Coded Aperture Radiation Detectors using a Bayesian Approach
}

\author{
Kyle Miller ${ }^{\mathrm{a}, *}$, Peter Huggins ${ }^{\mathrm{a}}$, Simon Labov ${ }^{\mathrm{b}}$, Karl Nelson ${ }^{\mathrm{b}}$, \\ Artur Dubrawski ${ }^{\mathrm{a}}$ \\ ${ }^{a}$ Auton Lab, the Robotics Institute, Carnegie Mellon University, 5000 Forbes Avenue, \\ Pittsburgh, PA 15213, USA. \\ ${ }^{b}$ Lawrence Livermore National Laboratory, Livermore, CA, USA.
}

\begin{abstract}
We investigate tradeoffs arising from the use of coded aperture gamma-ray spectrometry to detect and localize sources of harmful radiation in the presence of noisy background. Using an example application scenario of area monitoring and search, we empirically evaluate weakly supervised spectral, spatial, and hybrid spatio-spectral algorithms for scoring individual observations, and two alternative methods of fusing evidence obtained from multiple observations. Results of our experiments confirm the intuition that directional information provided by spectrometers masked with coded aperture enables gains in source localization accuracy, but at the expense of reduced probability of detection. Losses in detection performance can however be to a substantial extent reclaimed by using our new spatial and spatio-spectral scoring methods which rely on realistic assumptions regarding masking and its impact on measured photon distributions.
\end{abstract}

Keywords: coded aperture, wide area nuclear detection, distributed sensor networks

\section{Introduction}

Searching for nuclear and radiological threats with mobile radiation detectors remains problematic. In typical search operations, the environment is cluttered with benign nuisance radiation sources and large fluctuations in the natural radiation background [26]. Detecting sources of interest through this clutter requires discrimination. Two common methods of providing that discrimination are spectral and spatial. Spectral discrimination takes advantage of the

\footnotetext{
* Corresponding author

Email address: mille856@andrew.cmu.edu (Kyle Miller)

${ }^{1}$ This work has been partially supported by the U.S. Department of Energy under grant DE-NA0001736 and by the National Science Foundation under award 1320347. Lawrence Livermore National Laboratory is operated by Lawrence Livermore National Security, LLC, for the U.S. Department of Energy, National Nuclear Security Administration under Contract DE-AC52-07NA27344
}

Preprint submitted to Elsevier

September 12, 2016

(C) 2016. This manuscript version is made available under the Elsevier user license http://www.elsevier.com/open-access/userlicense/1.0/ 
difference in energy emissions between the sources of interest, and the background and common nuisance sources. This method has been shown to be very effective for most threatening sources, with some degradation in performance for sources that are highly shielded and/or lack significant spectral features, or that have features very similar to the background $[3,1]$. Spatial discrimination exploits the fact that most of the background fluctuations are due to large-scale features such as buildings and other structures, and changes in the composition of nearby materials, whereas the sources of interest are generally more compact [28]. Spatial discrimination has been quite successful as well, but suffers from inefficiencies in the imaging systems, and - not considering spectral information - cannot dismiss nuisance point sources that have spectral fingerprints that differ from source of interest [25, 24].

Evaluations of spectral and spatial orientated detection methodologies has been somewhat mixed $[28,23,5,6]$. Some studies have shown improved performance using imaging systems [23]. Others have shown that detection performance suffers when using imaging technology such as coded apertures [5]. The size and energy resolution of the detector have been implicated in determining relative performance of different systems [6].

In this paper we explore both spectral and spatial methods together to determine if a combination of these methods might be effective in the context of coded aperture gamma-ray spectrometers. We specifically aim to understand the empirical impact of using a coded aperture on detection performance. We use measurement based simulations to test the performance of detectors with modest spectral resolution (NaI scintillators) both with and without a coded aperture uniform redundant mask providing imaging capability. We analyze the data using two fusion methods, an analytic weighted projection, and Bayesian Aggregation [22] of evidence from multiple spectral measurements. We also consider alternative, weakly supervised methods of scoring individual observations that rely on spectral, spatial, and spatio-spectral analysis. We then compare and contrast the performance, highlighting the impact of choice of detection algorithm on source detectability and accuracy of its localization.

We demonstrate that naïvely applying a spectral method to a detector outfitted with a coded aperture results in a decrease in both threat detection and localization performance, as expected. Further, we show that our spatial method (with mask) is inferior to the spectral method (without mask) in detection, but superior in localization. Finally, we show that using a hybrid approach much of the detection performance lost by applying the coded aperture can be recovered while preserving the superior localization capabilities.

Section 2 details our methodology and the use of data, and detection algorithms. Section 3 describes experimental results, and Section 4 details our interpretations of the results and implications.

\section{Methods}

We compare different approaches by quantifying their performance in a simulated roadside threat detection scenario. We model this scenario on the 
RadMAP mobile detection unit [15, 20]: a vehicle outfitted with a $10 \times 10$ array of NaI detectors with coded aperture, GPS/IMU (Magellan ADU5) unit, 21 HPGe detectors, LiDAR system, and camera designed to traverse public roads and monitor the local area for radiological threats. We create a simulation environment that faithfully approximates the operation of such a vehicle save for the following simplifications. We use only the NaI detector array and GPS/IMU unit, ignoring the additional sensing equipment. We consider a flat environment, ignoring variations in altitude, pitch, and yaw resulting from uneven terrain. We disregard systematic differences in the data generating process caused by detector element position. In reality, detector elements experience some variation in photon counts as a function of their position in the array (e.g. closer to the ground, etc.). We believe that these simplifications will not have an appreciable effect on the comparison of the methods considered.

From empirical data collected by the RadMAP project [20], we reconstruct a detection vehicle path identifying the position, orientation, velocity, and time of each observation. From the observed photon measurements, we construct a generative background radiation model for the two operating conditions: with and without the coded aperture in place. Simulation replicates are conducted by sampling background measurements from the generative model and then drawing additional photon counts from a source model representing a point source with randomly generated position and intensity. For each detection algorithm, we find the point that maximizes the detection score in a neighborhood of $\pm 10 \mathrm{~m}$ surrounding the true source location both with and without the injected source counts. All observations within a horizon of $40 \mathrm{~m}$ from the source neighborhood are aggregated during scoring. Finally, the maximal detection scores along with their relative positions are analyzed to characterize detection and localization performance. We detail the simulation implementation below.

\subsection{Data and Detector Description}

The RadMAP data [20] set represents the observations of the MISTI mobile detection vehicle [15] traveling the roads of West Berkeley, Downtown Berkeley, South Side, and Solano California for four hours on May 1, 2012 from 9:25am to $1: 45 \mathrm{pm}$. Radiological data was stored in the list mode, recording time, energy, and photon count. Total background detected by the NaI array with coded aperture in place averaged approximately 10,000 photon counts per second. Positional data was measured at a frequency of $5 \mathrm{~Hz}$ recording time, latitude, longitude, altitude, pitch, and yaw. Average vehicle velocity was $5.4 \mathrm{~m} / \mathrm{s}$. Figure 1 shows the spatial layout of the path of the detection vehicle represented in this data set. Observations are represented as black points. The shaded gray region represents the space of candidate source locations.

The HPGe detector data was not used in this study and altitude, pitch, and yaw were ignored, imposing a perfectly flat landscape. The NaI sensor data were processed by first removing detection events with energy levels less than $30 \mathrm{keV}$. Remaining event-list data were converted to spectra with a 1s live time and 128 quadratically spaced energy bins. A live time of 1 s was judged to provide

the best compromise between spectral and temporal resolution. Quadratic bin 


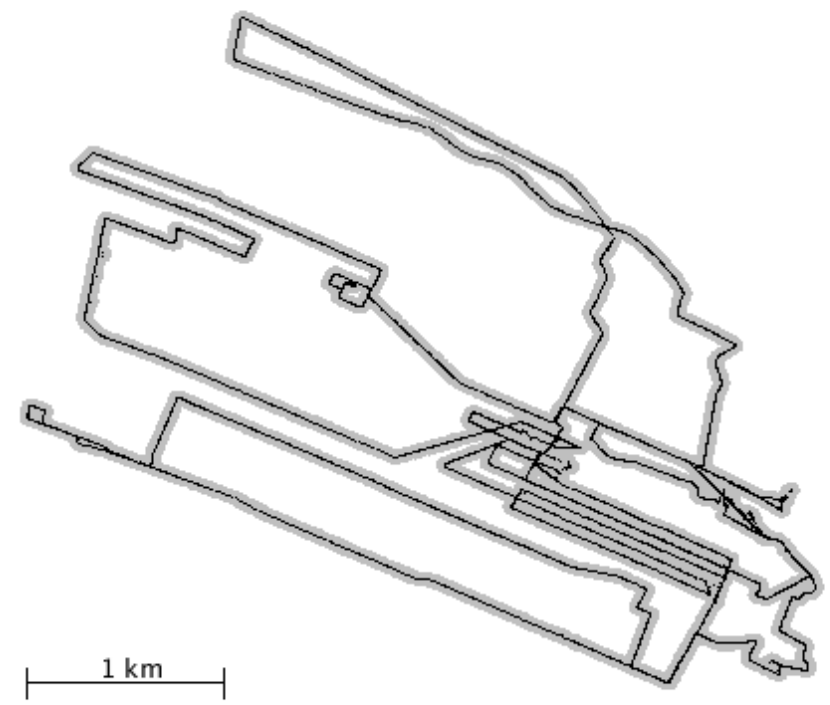

Figure 1: Path of detection vehicle in empirical RadMAP data. Black points represent observations, shaded gray region shows a $40 \mathrm{~m}$ envelope in which synthetic sources are placed during simulation. 
spacing greatly reduces spectrum size and processing costs, while preserving much of the useful energy resolution. Latitude and longitude were linearly interpolated to align with the middle of the spectral integration live time.

The $100 \mathrm{NaI}$ scintillators on the detection vehicle were arranged in a $10 \times 10$ grid. The coded aperture was placed $0.4 \mathrm{~m}$ in front of the detector array [15]. Each detector element measured $0.1 \mathrm{~m}$ in both height and width. For the purposes of simulation we suppose a detector of infinitesimal thickness. Integrated over a 1s live time, each empirical observation can be represented as a $128 \times 100$ array, each entry corresponding to one of the 128 energy channels and 100 detector elements.

\subsection{Synthetic Observation Generation}

The empirical data were used to learn a generative model of background radiation for the purposes of generating synthetic observations while maintaining systematic background variation. We take $E$ to represent an $100 N \times 128$ matrix of empirical photon counts wherein each row is an observation for a single detector element and each column is an energy bin. It is assumed that background observations are Poisson and $\boldsymbol{\lambda}^{\{i\}}$ represents the vector of empirical Poisson rates at the location of measurement $i=1 \ldots N$. We estimate $\boldsymbol{\lambda}^{\{i\}}$ as

$$
\begin{aligned}
\boldsymbol{\lambda}^{\{i\}}=\underset{\boldsymbol{\lambda}}{\operatorname{argmax}} & -\frac{1}{2}(\boldsymbol{\lambda}-\boldsymbol{\mu})^{T} K^{-1}(\boldsymbol{\lambda}-\boldsymbol{\mu}) \\
& +\mathbf{1}^{T} E_{d_{i}<10, .} \ln \boldsymbol{\lambda},
\end{aligned}
$$

where $\boldsymbol{\mu}$ and $K$ represent the global mean and covariance of the empirical data, 1 represents a vectors of ones, and $E_{d_{i}<10, \text {. represents the observations within }}$ $10 \mathrm{~m}$ of observation $i$. This corresponds to finding the $\boldsymbol{\lambda}$ that maximizes the likelihood of local data under a Poisson model with a Gaussian prior on the rates, which is conceptually similar to [14]. During simulation time, synthetic background observations are generated by taking a Poisson draw from each $\boldsymbol{\lambda}^{\{i\}}$. In the absence of the coded aperture, background exposure is slightly higher. We adjust the learned background model for the unmasked configuration by inflating the $\boldsymbol{\lambda}^{\{i\}}$ by a factor of $1 /(1-\xi)$, where $\xi$ represents the proportion of sight lines obscured by the coded aperture averaged over the detector elements. This factor corresponds to about a $13 \%$ increase in background rate over the masked configuration.

During simulation, the location of a single injected source is sampled uniformly from the set of locations that fall within $40 \mathrm{~m}$ of at least one observation taken enroute. The vertical position of the source and lower edge of the detector in all measurements is assumed to be zero. Further, we assume a point source of infinitesimal size. The exposure to a point source experienced by a detector element is calculated as the flux through the projection of the element onto a the unit sphere centered at the source.

We will now take a moment to detail the calculation of the exposure a detector element experiences from an arbitrary point source. We calculate the 
flux by first defining the helper function $h$

$$
\begin{aligned}
& h(x, y, z)=\int_{\operatorname{atan} 2(y, x)}^{\frac{\pi}{2}} \int_{\frac{\pi}{2}}^{\phi(\theta)} \sin \phi d \phi d \theta \\
& =\operatorname{sign}(x) \operatorname{atan}\left(\sqrt{\frac{x^{2} z^{2}}{x^{2} y^{2}+y^{4}+y^{2} z^{2}}}\right) .
\end{aligned}
$$

This function can be thought of as calculating the area of the projection of a rectangle with lower left corner at $(0, y, 0)$ and upper right corner at $(x, y, z)$ onto the unit sphere centered at the origin, where $y$ is the transverse distance between the detector and the source. We assume the source is positioned at the origin and detector element $i=1, \ldots, 100$ is described by lower left corner $\left(x_{i}, y, z_{i}\right)$ and upper right corner $\left(x_{i}+1, y, z_{i}+1\right)$. Then, the instantaneous exposure to a source at position $(x, y, z)$ is given by

$$
\begin{aligned}
\boldsymbol{e}_{i}^{\prime}(x, y, z)=\frac{1}{4 \pi} & {\left[h\left(x-x_{i+1}, y, z-z_{i+1}\right)+\right.} \\
& h\left(x-x_{i}, y, z-z_{i}\right)- \\
& h\left(x-x_{i+1}, y, z-z_{i}\right)- \\
& \left.h\left(x-x_{i}, y, z-z_{i+1}\right)\right] .
\end{aligned}
$$

Finally, exposure to a hypothetical source experienced in observation $k$ is calculated by integrating over the relative path of motion giving

$$
\boldsymbol{e}_{i}^{\{k\}}(x, y, 0)=\int_{0}^{\Delta t^{\{k\}}} e_{i}^{\prime}\left(x_{i}^{\{k\}}(t), y_{i}^{\{k\}}(t), 0\right) d t,
$$

where $\Delta t^{\{k\}}$ is the integration time and $\left(x_{i}^{\{k\}}(t), y_{i}^{\{k\}}(t)\right)$ describes the position of the source relative to moving detector. During simulation, this final integral is calculated numerically using the trapezoid rule. Vehicle path is approximated by straight line interpolation between measurement positions. Exposure for each element of the masked detector is calculated by first calculating the exposure of the intersection of the element with each mask element projected onto the plane of the detector. This can be easily calculated using (4) since the projection of each mask element will be rectangular and thus the intersection will be as well. Finally, the sum of the mask element exposures is subtracted from the unmasked exposure.

Again, for each detector element, the proportion of sight lines obscured by the coded aperture is calculated by integrating (3) over the surface of the coded aperture. This value was then averaged over detector elements to obtain $\xi$.

Photon counts due to source are injected into element $i$ observation $k$ by taking a Poisson draw with rate vector $\epsilon l_{s} e_{i}^{\{k\}} \boldsymbol{\lambda}_{s}$, where $\epsilon$ is the detector efficiency, $l_{s}$ is the source intensity, and $\boldsymbol{\lambda}_{s}$ is the source spectrum. In these simulations we take $\epsilon=0.6$. The exposure is either the masked or unmasked value depending on the configuration of the simulation. 


\subsection{Source Template}

For these experiments an artificial source spectrum was created to be a highfidelity representative of a shielded nuclear material under the assumption of a single low energy peak. The source peak somewhat overlaps the potassium peak in natural background, but is not aligned with it. Source intensities where chosen in a range that would give interesting comparisons, neither too weak to be undetectable nor too strong to give near perfect detection performance.

\subsection{Inference}

Detection and localization of radioactive sources is approached using two scoring algorithms: a well-established Censored Energy Windowing (CEW) and a proposed here for the fist time Pseudo-inverse Decoding (PID) for the unmasked and masked detectors respectively. These algorithms represent weakly informed detection methods, as they assume knowledge of some binary partition of energy channels that aid in source detection. We denote the set of energy channels in which source presence is high as $\mathcal{W}$. To provide the best comparison, the optimal signal-to-noise ratio (SNR) partition given our synthetic source design is used in our simulations. Apart from this partition however, the only other source specific information exploited is that the source is compact and immobile.

Hence, $\mathcal{W}$ is the set of not necessarily contiguous energy bins that jointly maximize the SNR. If $\boldsymbol{\lambda}_{\boldsymbol{b}}$ and $\boldsymbol{\lambda}_{\boldsymbol{s}}$ are the background and source spectra respectively, then $\operatorname{SNR}(\mathcal{W})=\sum_{i \in \mathcal{W}} \boldsymbol{\lambda}_{\boldsymbol{s}, i} / \sqrt{\sum_{i \in \mathcal{W}} \boldsymbol{\lambda}_{\boldsymbol{b}, i}}$. It is known that $\operatorname{SNR}(\mathcal{W})$ satisfies the linear time subset scan property [16]. So, $\mathcal{W}$ can be obtained quickly by first sorting the energy bins by the source to background ratio in descending order. Then, we take $\mathcal{W}$ to be the first $k$ bins, where $k$ gives the best value for the SNR over choices of the partition.

Additionally, we investigate two approaches for aggregating multiple individual scores. These are Bayesian Aggregation (BA) and Weighted Combining (WC), described below. The scoring algorithms take a single observation and a hypothetical source location and return a numeric score. The aggregating algorithms fuse scores obtained from multiple observations, producing a spatial map of aggregate source presence likelihood scores over which the location and value of the maximal score can be determined.

\subsubsection{Censored Energy Window}

The Censored Energy Window approach [18] assumes that under the null hypothesis (no source present) there is a linear relationship between the counts inside the source type specific energy window $\mathcal{W}$ and those outside $\mathcal{W}$. Thus, we fit a linear model of the form

$$
\sum_{i \in \mathcal{W}} Y_{., i}=Y_{., i \notin \mathcal{W} \boldsymbol{\beta},}
$$

where $Y_{k, i}$ represents the photon count for observation $k$ energy bin $i$. The model coefficients $\boldsymbol{\beta}$ are fit using the least squares approach. We generated 
additional synthetic background data to fit $\boldsymbol{\beta}$ prior to simulation. Then, for an observation the Censored Energy Window score is given as the signal to noise ratio:

$$
s=\frac{\sum_{i \in \mathcal{W}} Y_{., i}-Y_{., i \notin \mathcal{W} \boldsymbol{\beta}}}{\sqrt{Y_{., i \notin \mathcal{W} \boldsymbol{\beta}}}} .
$$

\subsubsection{Pseudo-inverse Decoding}

For a detector array equipped with a coded aperture, each of the 100 detector elements has a different field of view. As a result, one can write the the photon intensity each detector element experiences as a linear combination of the intensities of world-pixels; regions of the field of view for which intensity is presumed constant. If $\boldsymbol{y}$ respresents the observed data and $\boldsymbol{x}$ represents the world-pixels, suitably unraveled into a vector, the $\mathrm{E}[\boldsymbol{y}]=A \boldsymbol{x}$ for some aperture matix $A$ encoding the exposures. By means of various inversion strategies $\boldsymbol{x}$ can be reconstructed from $\boldsymbol{y}$ yielding an image of gamma intensity $[7,9,27,11]$.

We take a similar to decoding data collected with a coded aperture, although we do not seek to reconstruct an image. Instead we imagine the field of view to be more or less constant in intensity with a single hot spot (i.e. the target source). Our approach is basically to test the quality of the fit of such assumptions. If we suppose the total photon count within a fixed energy window $\mathcal{W}$ is Gaussian in the background and source rates with constant variance. Further, we impose a Gaussian prior on the estimate of the background rate. Under this model, the best estimate of background $b$ and source $s$ is given by the least squares solution to the linear system

$$
\left[\begin{array}{c}
\boldsymbol{y} \\
\frac{\mu_{b} \sigma}{\sigma_{b}}
\end{array}\right]=\left[\begin{array}{cc}
\mathbf{1} & \boldsymbol{e} \\
\frac{\sigma}{\sigma_{b}} & 0
\end{array}\right]\left[\begin{array}{l}
b \\
s
\end{array}\right] .
$$

Here, $\boldsymbol{y}, \boldsymbol{e}$, and $\mathbf{1}$ are 100 dimensional vectors of detector element counts, element specific exposures, and ones respectively. The parameters $\mu_{b}, \sigma_{b}$, and $\sigma$ encode the background prior mean and standard deviation, and the standard deviation of the observations $\boldsymbol{y}_{i}$. Solving for $s$ gives

$$
s=\frac{\boldsymbol{\alpha}^{\top} \boldsymbol{y}}{\boldsymbol{\alpha}^{\top} \boldsymbol{e}}-\frac{\boldsymbol{\alpha}^{\top} \mathbf{1}}{\boldsymbol{\alpha}^{\top} \boldsymbol{e}} \mu_{b}
$$

where $\boldsymbol{\alpha}=\left(100+\sigma^{2} / \sigma_{b}^{2}\right) \boldsymbol{e}-\boldsymbol{e}^{\boldsymbol{\top}} \mathbf{1 1}$. If $\mathrm{E}[\boldsymbol{y}]=\operatorname{Var}[\boldsymbol{y}]=\mathbf{1} \mu_{b}^{\star}+$ le where $\mu_{b}^{\star}$ is the true in-window mean background and $l$ is the true in-window source intensity, then $\mathrm{E}[s]=l-\frac{\boldsymbol{\alpha}^{\top} \mathbf{1}}{\boldsymbol{\alpha}^{\top} \boldsymbol{e}}\left(\mu_{b}^{\star}-\mu_{b}\right)$ and $\operatorname{Var}[s]=\frac{\boldsymbol{\alpha}^{\top} \boldsymbol{\alpha}}{\left(\boldsymbol{\alpha}^{\top} \boldsymbol{e}\right)^{2}} \mu_{b}^{\star}+\frac{(\boldsymbol{\alpha} \circ \boldsymbol{\alpha})^{\top} \boldsymbol{e}}{\left(\boldsymbol{\alpha}^{\top} \boldsymbol{e}\right)^{2}} l$. Since (8) is a linear combination of many independent Poisson random variables $\boldsymbol{y}, s$ will be well approximated by a Gaussian distribution. The KullbackLeibler (KL) divergence between the distributions of $s$ with and without a source present is given by $\frac{\left(\boldsymbol{\alpha}^{\top} \boldsymbol{e}\right)^{2}}{2 \boldsymbol{\alpha}^{\top} \boldsymbol{\alpha}} \frac{l^{2}}{\mu_{b}^{\star}}+\frac{(\boldsymbol{\alpha} \circ \boldsymbol{\alpha})^{\top} \boldsymbol{e}}{2 \boldsymbol{\alpha}^{\top} \boldsymbol{\alpha}} \frac{l}{\mu_{b}^{\star}}-\frac{1}{2} \ln \left(\frac{(\boldsymbol{\alpha} \circ \boldsymbol{\alpha})^{\top} \boldsymbol{e}}{\boldsymbol{\alpha}^{\top} \boldsymbol{\alpha}} \frac{l}{\mu_{b}^{\star}}+1\right)$ temporarily assuming $\mu_{b}=\mu_{b}^{\star}$. This expression is dominated by the first term, so choosing $\mathcal{W}$ that maximizes the SNR approximately maximizes the KL-divergence between source-present and source-absent score distributions. Additionally, this choice 
of $\mathcal{W}$ depends only on source and background spectral shape, not on context dependent specifics such as $\boldsymbol{\alpha}$ or the source strength. Therefore we choose the $\mathcal{W}$ that maximizes the SNR, as in CEW.

It remains to choose values for $\mu_{b}, \sigma_{b}$, and $\sigma$. A natural choice is to use global estimates assuming no source. Alternatively, one can use local estimates which one might hope will capture local background variation. The maximum likelihood estimate for the local in-window mean and variance is the in-window counts averaged over the detector elements, i.e. we take $\sigma^{2}=\sigma_{b}^{2}=\mu_{b}=\frac{1}{100} \mathbf{1}^{\top} \boldsymbol{y}$. This causes (8) to become

$$
s=\frac{1}{\boldsymbol{\alpha}^{\top} \boldsymbol{e}}\left(\boldsymbol{\alpha}-\frac{1}{100} \boldsymbol{\alpha}^{\boldsymbol{\top}} \mathbf{1 1}\right)^{\top} \boldsymbol{y} .
$$

As a consequence we incur some bias in exchange for reduced variance, i.e.

$$
\begin{aligned}
\mathrm{E}[s] & =\left(1-\frac{\boldsymbol{\alpha}^{\boldsymbol{\top}} \mathbf{1} \boldsymbol{e}^{\boldsymbol{\top}} \mathbf{1}}{100 \boldsymbol{\alpha}^{\top} \boldsymbol{e}}\right) l, \\
\operatorname{Var}[s] & =\frac{1}{\left(\boldsymbol{\alpha}^{\top} e\right)^{2}} \sum_{i}\left(\boldsymbol{\alpha}_{i}-\frac{1}{100} \boldsymbol{\alpha}^{\boldsymbol{\top}} \mathbf{1}\right)^{2}\left(\mu_{b}^{\star}+e_{i} l\right) .
\end{aligned}
$$

It turns out to be a good trade in this application. This modification alters some of the coefficients of the KL-divergence expression, but since SNR is insensitive to multiplicative factors, we maintain the same window $\mathcal{W}$ as above. We refer to this method as PID for Pseudo-inverse Decoding.

Another alternative design choice is to introduce a linear regression model in the spirit of CEW, as the prior $\mu_{b}$. That is, we take $\sigma^{2}=\mu_{b}=Y_{., i \notin \mathcal{W} \boldsymbol{\beta}}$ and estimate of the standard deviation of the residual $\sigma_{b}$ during training. This gives a straightforward hybrid spectral-spatial method, which we refer to as PIDCEW. For simplicity we retain the same choice of $\mathcal{W}$ as before.

\subsubsection{Bayesian Aggregation}

Bayesian aggregation (BA) [22] is used to combine information from multiple measurements to produce a single score for source presence as a function of location. BA is applied to both CEW and PID in the same fashion. Given a set of scores $\left\{s^{\{k\}}\right\}_{k=1}^{n}$ generated from $n$ observations supposing a source at position $(x, y)$, we calculate the likelihood of observing such a score $P\left(s^{\{k\}} \mid l_{j}, \boldsymbol{y}^{\{k\}}, \boldsymbol{e}^{\{k\}}\right)$ for each of a finite set of hypothetical source intensities $l_{j}, j=1, \ldots, m$. Here $y^{\{k\}}$ represents either, for observation $k$, the observed counts summed over energy bins in $\mathcal{W}$ for PID or observed counts summed over detector elements for CEW. In these simulations, we used 20 quadratically spaced source intensity hypotheses from 1 to $1000 \mu \mathrm{Ci}$. We then compute the source-to-no-source likelihood ratio and marginalize over hypothesized source intensity as

$$
\operatorname{BA}(x, y)=\sum_{j=1}^{m} \prod_{k=1}^{n} \frac{P\left(s^{\{k\}} \mid l_{j}, \boldsymbol{y}^{\{k\}}, e^{\{k\}}(x, y)\right)}{P\left(s^{\{k\}} \mid 0, \boldsymbol{y}^{\{k\}}, \mathbf{0}\right)} .
$$

In this formulation we can see the rationale for imposing a horizon on the consideration set of measurements. Measurements taken at a large distance 
from the source will have very low exposure resulting in a likelihood ratio very close to 1.

The PID score is a linear combination of many independent random variables and is well approximated by the Gaussian distribution. We have empirically verified that this approximation holds for our simulations. We therefore take $P(s \mid l, \boldsymbol{y}, \boldsymbol{e})=P\left((\boldsymbol{\alpha}-\mathbf{1} \bar{\alpha})^{\top} \boldsymbol{y} \mid l, \boldsymbol{e}\right)$ as Gaussian with mean and variance given in (10) and (11) using $\mu_{b}^{\star}=\frac{1}{100} \boldsymbol{y}^{\top} \mathbf{1}$. For PIDCEW, we approximate the Gaussian parameters using mean $l$ and variance as PID plus the variance due to the residual, which is determined during model estimation without source present. Further, the variance estimate of the residual is conditioned on the total out of window counts $\bar{y}$ as described shortly.

For the CEW decoding method, the score $s$ can be viewed as the ratio of two random variables. We apply the Geary-Hinkley [8] transform to convert $s$ to a standard normal random variable. To effect the transformation, we take the residual counts (numerator of $s$ ) to have mean $\epsilon \boldsymbol{l}^{\boldsymbol{\top}} \mathbf{1}+\hat{\mu}_{1}(\bar{y})$ and variance $\epsilon l \mathbf{1}^{\top} \boldsymbol{e}+\hat{\sigma}_{1}^{2}(\bar{y})$, where $\hat{\mu}_{1}$ and $\hat{\sigma}_{1}^{2}$ are functions learned from training data that model mean and variance under the null hypothesis of no presence of a source as a function of out-of-window counts $\bar{y}$. Similarly, we take the square root of the predicted counts (denominator of $s$ ) to have mean $\hat{\mu}_{2}(\bar{y})$ and $\hat{\sigma}_{2}^{2}(\bar{y})$. Finally, we naïvely assume the numerator and denominator to be uncorrelated.

The regression functions for estimating the residual variance in PIDCEW and the mean and variance of the numerator and denominators for the GearyHinkley transform are fit at the same time as the regression models are learned using training data without source present. These values are taken as functions of $\bar{y}$ with represents either the total counts for PIDCEW or the total out-ofwindow counts for CEW. We use a trend filtering [10] approach that penalizes the fit according to the magnitude of the second derivative. Since $\bar{y}^{\{k\}}$ takes only integer values, we use training data to seek vectors $\boldsymbol{v}^{\star}$ and $\boldsymbol{u}^{\star}$ indexed by total count value $\bar{y}$ that satisfy the system of optimization problems

$$
\begin{aligned}
\boldsymbol{v}^{\star} & =\underset{\boldsymbol{v}}{\operatorname{argmin}} \sum_{i} \frac{1}{2 \boldsymbol{u}_{\bar{y}^{\{i\}}}}\left(\boldsymbol{v}_{\bar{y}^{\{i\}}}-\boldsymbol{y}_{i}\right)^{2} \\
& +\lambda_{v}\|D \boldsymbol{v}\|_{2}^{2}, \\
\boldsymbol{u}^{\star} & =\underset{\boldsymbol{u}}{\operatorname{argmin}} \sum_{i} \frac{1}{2}\left[\boldsymbol{u}_{\bar{y}^{\{i\}}}-\left(\boldsymbol{v}_{\bar{y}^{\{i\}}}-\boldsymbol{y}_{i}\right)^{2}\right]^{2} \\
& +\lambda_{u}\|D \boldsymbol{u}\|_{2}^{2},
\end{aligned}
$$

where $D$ is a sparse matrix encoding a second order finite-difference method for the second derivative and $\boldsymbol{y}_{i}$ represents the $i^{\text {th }}$ value of numerator or denominator where appropriate. We then define $\hat{\mu}_{1}(\bar{y})=\boldsymbol{v}_{\bar{y}}^{\star}$ when $\min _{i}\left(\bar{y}^{\{i\}}\right) \leq \bar{y} \leq$ $\max _{i}\left(\bar{y}^{\{i\}}\right)$, and take $\boldsymbol{v}_{\min _{i}\left(\bar{y}^{\{i\}}\right)}$ or $\boldsymbol{v}_{\max _{i}\left(\bar{y}^{\{i\}}\right)}$ if $\bar{y}$ falls outside the range of the training data. Similarly, we define $\hat{\sigma}_{1}^{2}(\bar{y})=\boldsymbol{u}_{\bar{y}}^{\star}$ clamping indices to the range observed in training. The remaining mean and variance functions are are calculated in the same fashion, using the appropriate values for the $\boldsymbol{y}_{i}$. Fixing $\boldsymbol{u}$ and setting the gradient to zero in (13) results in a linear system. Similarly fixing 
$\boldsymbol{v}$ yields a linear system in (14). We initialize $\boldsymbol{v}_{\bar{y}}$ and $\boldsymbol{u}_{\bar{y}}$ to global estimates of mean and variance respectively for all $\bar{y}$ and alternate solving problems (13) and (14) until convergence. Note that summing over observations $i$ and dividing by the variance $\boldsymbol{u}$ in the case of (13) biases the optimization to remain closer to the local empirical mean or variance, respectively, in regions with higher data support and/or lower variance, possibly sacrificing agreement with the local estimates in other regions for the sake of smoothness.

\subsubsection{Weighted Combining}

Weighted combining is an analytic weighted projection method for aggregating multiple measurements [17]. To account for the presence of the mask and movement of the detection vehicle, we replace the typical weighting factor of reciprocal squared distance $\left(\frac{1}{r^{2}}\right)$ [17] with the total detector exposure. For each measurement $k$, WC takes source $s_{c}^{\{k\}}$ and background $b^{\{k\}}$ count estimates, total exposure $\mathbf{1}^{\top} \boldsymbol{e}^{\{k\}}(x, y)$, and a hypothetical source location $(x, y)$. For CEW, the predicted window counts and the residual serve as estimates for background and source levels respectively. For PID, the estimate of $b$ and $s \mathbf{1}^{\top} \boldsymbol{e}$ do the same. The WC score is then calculated as the aggregate signal to noise ratio

$$
\mathrm{WC}(x, y)=\frac{\sum_{k}^{n} s_{c}^{\{k\}} \mathbf{1}^{\top} \boldsymbol{e}^{\{k\}}(x, y)}{\sqrt{\sum_{k}^{n} b^{\{k\}}\left(\mathbf{1}^{\top} \boldsymbol{e}^{\{k\}}(x, y)\right)^{2}}} .
$$

The weights for each measurement in this scheme are optimal in the sense that they give the linear combination that maximizes theoretical SNR, assuming given source exposure constants are accurate.

\section{Results}

The generative background model smooths local background variation using a boxcar filter of radius $10 \mathrm{~m}$. Table 1 shows a comparison of the total-counts in the empirical data with those expected under the learned generative model. The generative model smooths out the most extreme observations, but expected counts in the $10^{\text {th }}, 50^{\text {th }}$, and $90^{\text {th }}$ percentiles align fairly well with the empirical distribution. As a result, the simulation environment reflects significant variation in local background intensity, which is an important element of the threat detection problem in the real world $[28,26]$.

During simulation, source intensities were sampled uniformly from within the ranges of $10-25,50-75,100-250$, and $500-750 \mu \mathrm{Ci}$. Approximately 12,000 replicates were run for each method, combination of inference and aggregation algorithm, and source intensity range. Distance of closest approach (DOCA) was reasonably uniform over the interval of 1 to $40 \mathrm{~m}$, with a few samples further than $40 \mathrm{~m}$ from the detector. In our simulation framework, sources may be placed further than $40 \mathrm{~m}$ from the closest usable observation since we restrict to those observations in which the mask faces the source when performing inference but not when selecting locations. 
In Figure 2 we show Receiver Operating Characteristic (ROC) curves for all but the highest of the considered source intensities $(10-25 \mu \mathrm{Ci}, 50-75 \mu \mathrm{Ci}$, and $100-250 \mu \mathrm{Ci}$ ), for Bayesian aggregation (top row) and weighted combining (bottom row) fusion methods. Each graph depicts ROC performance of 4 scoring methods: CEW without masking (we expect it to upper-bound detection performance of algorithms applied on coded aperture data), and CEW, PID, and PIDCEW, all with coded aperture masking. In these plots, the true positive rate (TPR) is identical to probability of detection (PD) and it can be interpreted as the proportion of threat sources that were successfully detected by each method. The false positive rate $(\mathrm{FPR})$ can be interpreted as the proportion of $20 \times 20 \mathrm{~m}$ patches that generated false detections. Naïvely applying CEW to the masked detector results in a significant drop in probability of detection.

Table 2 shows the probability of detection at an FPR of 0.001 for each source intensity range, mask configuration, and algorithm, using BA. Table 3 shows the same for WC. The magnitude of the loss in detection performance when applying the coded aperture using CEW is correlated with source intensity: weaker sources lead to larger drops in performance. Enhancing the prior however, improves detection performance of PIDCEW compared to naïve CEW. While PIDCEW does not perform as well in detection as CEW without the coded aperture, it does recover much of the detection performance lost by $\mathrm{CEW}$ when the mask is applied. As with masked CEW, the performance gap relative to unmasked CEW is largest with weak sources. Yet detection performance drops precipitously for both PID and PIDCEW if the coded aperture is removed, demonstrating that the coded aperture is responsible for the a significant portion of the algorithm's capabilities. We also note that Bayesian Aggregation allows almost universally significantly better source detectability than weighted combining method in either masked or unmasked scenarios.

Graphs in Figure 3 represent the average probability of detection for each method and configuration presented in Figure 2 as a function of distance of closest approach. They characterize the effective detection range of each method. Weak sources $(10-25 \mu \mathrm{Ci})$ are effectively undetectable at a range of $30 \mathrm{~m}$, while stronger sources are effectively detected at all distances simulated. Detection capability as a function of DOCA follows overall detection performance trends, with unmasked CEW showing the best detection performance at the furthest distances, followed by PIDCEW with aperture.

To quantify localization performance it is necessary to define a detection event, since it is not appropriate to consider the localization of sources a method did not detect. Toward this end, we define a detection by fixing the FPR at $0.1 \%$. This yields different thresholds and different probabilities of detection for each method. We feel this approach is practical since it proposes to evaluate each method's performance under the constraint that they generate the same quantity of nuisance alerts (false detections). Additionally, determining source location by maximizing detection score over a $20 \times 20 \mathrm{~m}$ square centered on the true source location biases the estimate of average localization error. That is, it is not possible for a detector to localize worse than within the evaluation region. Thus, we report the proportion of detections for which the maximal score was 
Table 1: Total-count percentiles of empirical data and expected values under the learned generative model, with $95 \%$ confidence intervals. Intervals are computed using jackknife estimation [21].

\begin{tabular}{l|cc} 
& Empirical & Model (expected) \\
\hline $\min$ & $0.0 \pm<0.1$ & $50.7 \pm 0.1$ \\
$1 \%$ & $0.0 \pm<0.1$ & $60.2 \pm 1.0$ \\
$10 \%$ & $75.0 \pm<0.1$ & $80.2 \pm 0.4$ \\
$50 \%$ & $94.0 \pm<0.1$ & $95.0 \pm 0.3$ \\
$90 \%$ & $119.0 \pm<0.1$ & $113.1 \pm 0.7$ \\
$99 \%$ & $150.0 \pm<0.1$ & $135.9 \pm 0.9$ \\
$\max$ & $265.9 \pm 19.4$ & $170.1 \pm 8.0$
\end{tabular}

Table 2: Probability of detection at $0.1 \%$ FPR with $95 \%$ confidence intervals using Bayesian aggregation. Source intensity $(\mu \mathrm{Ci})$ is evenly distributed within the indicated ranges. The largest value in each row is represented in bold.

\begin{tabular}{cc|ccc} 
Aperture & Intensity & $\mathrm{CEW}(\mathrm{BA})$ & $\mathrm{PIDCEW}(\mathrm{BA})$ & $\mathrm{PID}(\mathrm{BA})$ \\
\hline absent & $10-25$ & $\mathbf{0 . 3 3 6} \pm \mathbf{0 . 0 1 2}$ & $0.324 \pm 0.012$ & $0.044 \pm 0.005$ \\
absent & $50-75$ & $\mathbf{0 . 7 4 3} \pm \mathbf{0 . 0 1 1}$ & $0.508 \pm 0.012$ & $0.118 \pm 0.008$ \\
absent & $100-250$ & $\mathbf{0 . 9 3 0} \pm \mathbf{0 . 0 0 6}$ & $0.568 \pm 0.012$ & $0.208 \pm 0.010$ \\
absent & $500-750$ & $\mathbf{0 . 9 8 7} \pm \mathbf{0 . 0 0 3}$ & $0.699 \pm 0.011$ & $0.387 \pm 0.012$ \\
\hline present & $10-25$ & $0.210 \pm 0.010$ & $\mathbf{0 . 2 3 3} \pm \mathbf{0 . 0 1 0}$ & $0.130 \pm 0.008$ \\
present & $50-75$ & $0.530 \pm 0.012$ & $\mathbf{0 . 6 4 1} \pm \mathbf{0 . 0 1 2}$ & $0.509 \pm 0.012$ \\
present & $100-250$ & $0.843 \pm 0.009$ & $\mathbf{0 . 9 0 3} \pm \mathbf{0 . 0 0 7}$ & $0.849 \pm 0.009$ \\
present & $500-750$ & $0.981 \pm 0.003$ & $\mathbf{0 . 9 8 5} \pm \mathbf{0 . 0 0 3}$ & $0.967 \pm 0.004$
\end{tabular}

less than $10 \mathrm{~m}$ from the source, and calculate mean error for these events only. This proportion can be thought of as the localization rate: the proportions of detections which can be said to have also been practically located. The mean error gives the expected error of detected and localized sources. Tables 4 (BA) and 6 (WC) give the average error given that a detection has been located to within $10 \mathrm{~m}$ of localization error, for each method and source intensity range. Tables $5(\mathrm{BA})$ and $7(\mathrm{WC})$ give the average probability that a detection is located to within $10 \mathrm{~m}$ of localization error, for each method and source intensity range. Figure 4 shows the average probability that error of source localization is within $10 \mathrm{~m}$ of the true location of the source, as a function of distance of closest approach for each method and configuration. The PID and PIDCEW methods with BA show superior likelihood to localize a detected source to within $10 \mathrm{~m}$. This is consistent across source intensities. PIDCEW with WC shows the same trend, while PID with WC fails at larger distances.

\section{Discussion}

We have investigated the impact of a coded aperture on a roadside nuclear threat detection scenario. Our simulation framework was designed to be reason- 


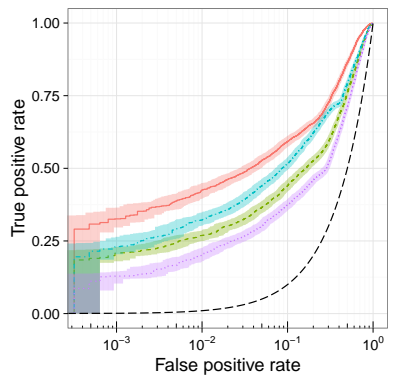

(a) $10-25 \mu \mathrm{Ci}(\mathrm{BA})$

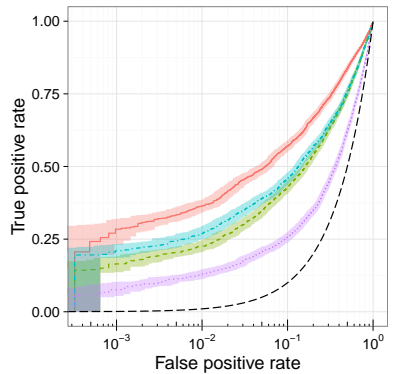

(d) $10-25 \mu \mathrm{Ci}(\mathrm{WC})$

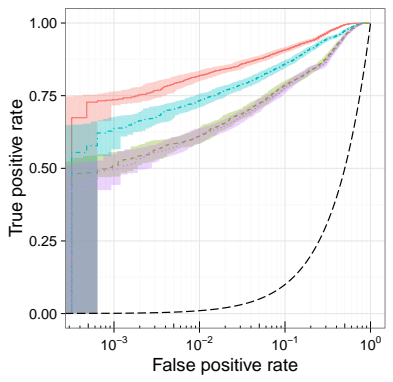

(b) $50-75 \mu \mathrm{Ci}(\mathrm{BA})$

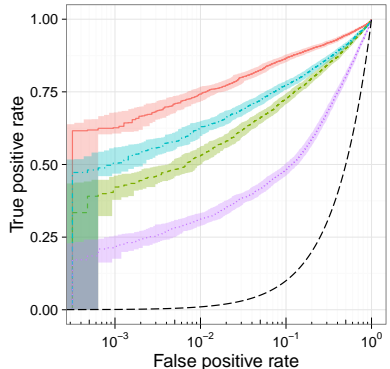

(e) $50-75 \mu \mathrm{Ci}(\mathrm{WC})$

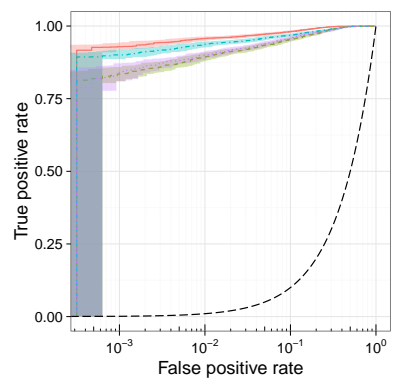

(c) $100-250 \mu \mathrm{Ci}(\mathrm{BA})$

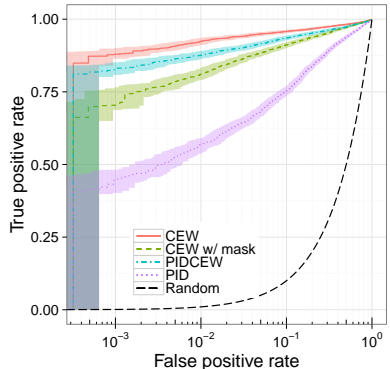

(f) $100-250 \mu \mathrm{Ci}(\mathrm{WC})$

Figure 2: Receiver operating characteristic (ROC) curves for each aggegration algorithm and the three lowest source intensity ranges. CEW without coded aperture shown in red (solid), CEW with coded aperture shown in green (dash), PIDCEW with aperture is shown in blue (dot), and PID with aperture shown in purple (dot-dash). Shaded regions correspond to $95 \%$ confidence intervals.

Table 3: Probability of detection at $0.1 \%$ FPR with $95 \%$ confidence intervals using weighted combining. Source intensity $(\mu \mathrm{Ci})$ is evenly distributed within the indicated ranges. The largest value in each row is represented in bold.

\begin{tabular}{cc|ccc} 
Aperture & Intensity & $\mathrm{CEW}(\mathrm{WC})$ & $\mathrm{PIDCEW}(\mathrm{WC})$ & $\mathrm{PID}(\mathrm{WC})$ \\
\hline absent & $10-25$ & $0.289 \pm 0.011$ & $\mathbf{0 . 3 1 8} \pm \mathbf{0 . 0 1 2}$ & $0.038 \pm 0.005$ \\
absent & $50-75$ & $0.643 \pm 0.012$ & $\mathbf{0 . 6 9 3} \pm \mathbf{0 . 0 1 1}$ & $0.083 \pm 0.007$ \\
absent & $100-250$ & $0.882 \pm 0.008$ & $\mathbf{0 . 8 9 6} \pm \mathbf{0 . 0 0 8}$ & $0.119 \pm 0.008$ \\
absent & $500-750$ & $0.980 \pm 0.003$ & $\mathbf{0 . 9 8 2} \pm \mathbf{0 . 0 0 3}$ & $0.168 \pm 0.009$ \\
\hline present & $10-25$ & $0.169 \pm 0.009$ & $\mathbf{0 . 2 1 3} \pm \mathbf{0 . 0 1 0}$ & $0.087 \pm 0.007$ \\
present & $50-75$ & $0.429 \pm 0.012$ & $\mathbf{0 . 5 1 6} \pm \mathbf{0 . 0 1 2}$ & $0.231 \pm 0.010$ \\
present & $100-250$ & $0.743 \pm 0.011$ & $\mathbf{0 . 8 3 2} \pm \mathbf{0 . 0 0 9}$ & $0.458 \pm 0.012$ \\
present & $500-750$ & $0.965 \pm 0.005$ & $\mathbf{0 . 9 8 1} \pm \mathbf{0 . 0 0 3}$ & $0.881 \pm 0.008$
\end{tabular}




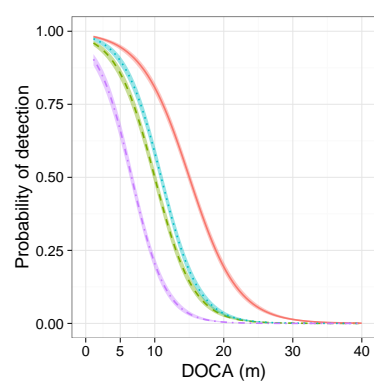

(a) $10-25 \mu \mathrm{Ci}(\mathrm{BA})$

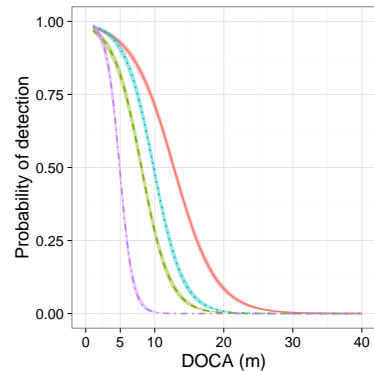

(d) $10-25 \mu \mathrm{Ci}(\mathrm{WC})$

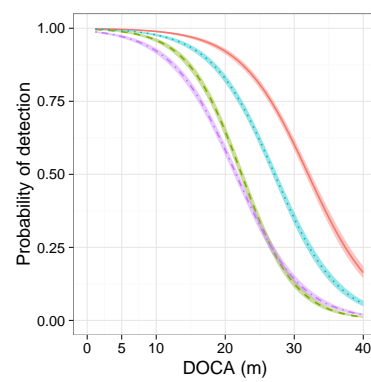

(b) $50-75 \mu \mathrm{Ci}(\mathrm{BA})$

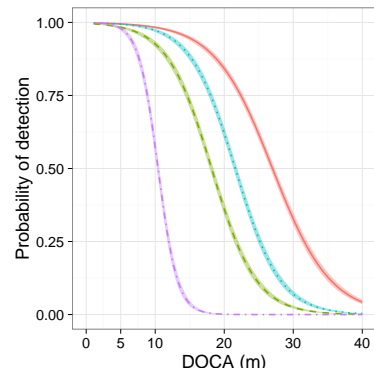

(e) $50-75 \mu \mathrm{Ci}(\mathrm{WC})$

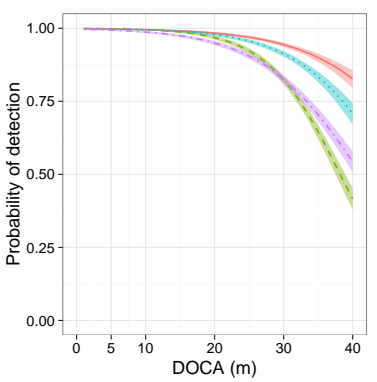

(c) $100-250 \mu \mathrm{Ci}(\mathrm{BA})$

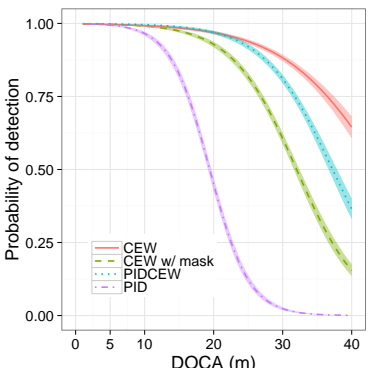

(f) $100-250 \mu \mathrm{Ci}(\mathrm{WC})$

Figure 3: Probability of detection as a function of distance of closest approach (DOCA) at $0.1 \%$ FPR for each aggregation algorithm and the three lowest source intensity ranges. CEW without coded aperture shown in red (solid), CEW with coded aperture shown in green (dash), PIDCEW with aperture is shown in blue (dot), and PID with aperture shown in purple (dot-dash). Shaded regions correspond to $95 \%$ confidence intervals.

Table 4: Mean localization error $(\mathrm{m})$ of detections made within the $10 \mathrm{~m}$ radius of the source at $0.1 \%$ FPR using Bayesian aggregation. Confidence reflects $95 \%$ intervals. Source intensity $(\mu \mathrm{Ci})$ is evenly distributed within the indicated ranges. The smallest error in each row is represented in bold.

\begin{tabular}{cc|ccc} 
Aperture & Intensity & CEW(BA) & PIDCEW(BA) & PID(BA) \\
absent & $10-25$ & $\mathbf{3 . 6 5} \pm \mathbf{0 . 1 2}$ & $4.99 \pm 0.13$ & $6.20 \pm 0.18$ \\
absent & $50-75$ & $\mathbf{2 . 9 7} \pm \mathbf{0 . 0 9}$ & $4.93 \pm 0.14$ & $5.00 \pm 0.16$ \\
absent & $100-250$ & $\mathbf{2 . 3 5} \pm \mathbf{0 . 0 7}$ & $5.11 \pm 0.13$ & $4.35 \pm 0.13$ \\
absent & $500-750$ & $\mathbf{1 . 4 3} \pm \mathbf{0 . 0 5}$ & $5.06 \pm 0.14$ & $3.97 \pm 0.11$ \\
\hline present & $10-25$ & $\mathbf{3 . 8 1} \pm \mathbf{0 . 1 4}$ & $4.04 \pm 0.10$ & $4.23 \pm 0.11$ \\
present & $50-75$ & $3.27 \pm 0.11$ & $\mathbf{2 . 5 9} \pm \mathbf{0 . 0 8}$ & $\mathbf{2 . 5 9} \pm \mathbf{0 . 0 8}$ \\
present & $100-250$ & $2.72 \pm 0.08$ & $1.62 \pm 0.05$ & $\mathbf{1 . 5 1} \pm \mathbf{0 . 0 5}$ \\
present & $500-750$ & $2.05 \pm 0.06$ & $1.33 \pm 0.04$ & $\mathbf{0 . 7 2} \pm \mathbf{0 . 0 3}$
\end{tabular}




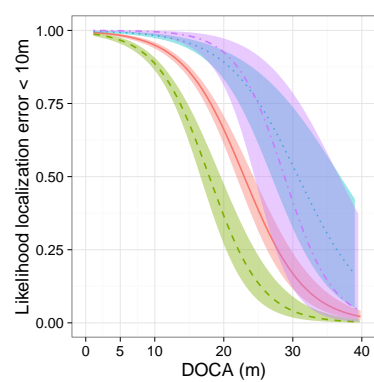

(a) $10-25 \mu \mathrm{Ci}(\mathrm{BA})$

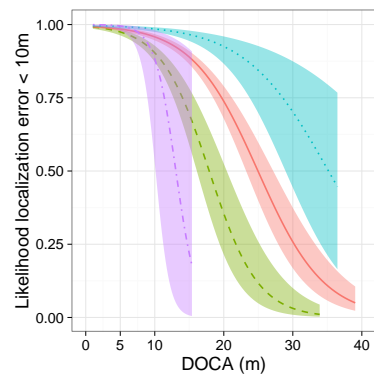

(d) $10-25 \mu \mathrm{Ci}(\mathrm{WC})$

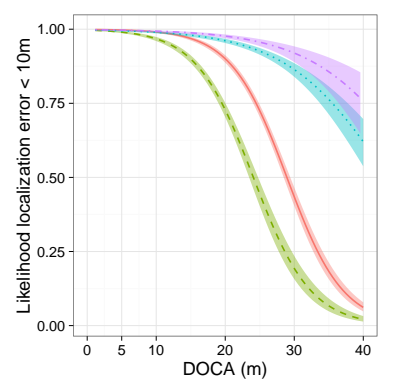

(b) $50-75 \mu \mathrm{Ci}(\mathrm{BA})$

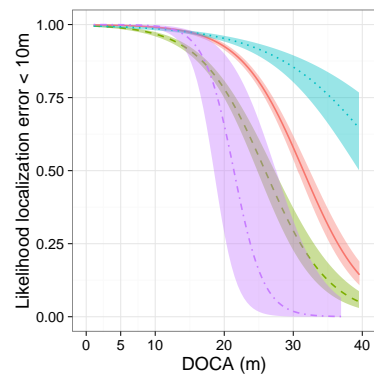

(e) $50-75 \mu \mathrm{Ci}(\mathrm{WC})$

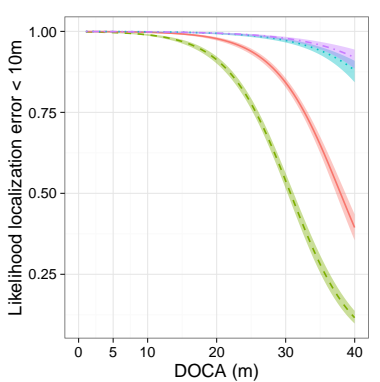

(c) $100-250 \mu \mathrm{Ci}(\mathrm{BA})$

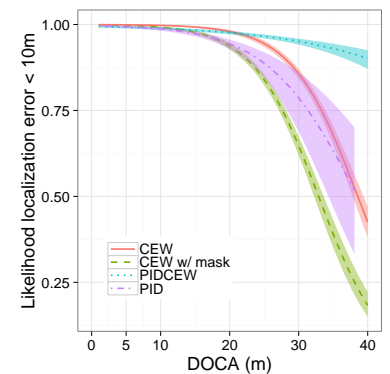

(f) $100-250 \mu \mathrm{Ci}(\mathrm{WC})$

Figure 4: Likelihood localization error is within $10 \mathrm{~m}$ as a function of distance of closest approach (DOCA) at $0.1 \%$ FPR for each aggregation algorithm and the three lowest source intensity ranges. CEW without coded aperture shown in red (solid), CEW with coded aperture shown in green (dash), PIDCEW with aperture is shown in blue (dot), and PID with aperture shown in purple (dot-dash). Shaded regions correspond to 95\% confidence intervals.

Table 5: Proportion of detections made within the $10 \mathrm{~m}$ radius of the source at $0.1 \%$ FPR using Bayesian aggregation. Confidence reflects $95 \%$ intervals. Source intensity $(\mu \mathrm{Ci})$ is evenly distributed within the indicated ranges. The largest proportion in each row is represented in bold.

\begin{tabular}{cc|ccc} 
Aperture & Intensity & CEW(BA) & PIDCEW(BA) & PID(BA) \\
absent & $10-25$ & $\mathbf{0 . 3 4} \pm \mathbf{0 . 0 1}$ & $0.32 \pm 0.01$ & $0.23 \pm 0.01$ \\
absent & $50-75$ & $\mathbf{0 . 6 4} \pm \mathbf{0 . 0 1}$ & $0.31 \pm 0.01$ & $0.29 \pm 0.01$ \\
absent & $100-250$ & $\mathbf{0 . 8 3} \pm \mathbf{0 . 0 1}$ & $0.29 \pm 0.01$ & $0.40 \pm 0.01$ \\
absent & $500-750$ & $\mathbf{0 . 9 6} \pm<\mathbf{0 . 0 1}$ & $0.25 \pm 0.01$ & $0.53 \pm 0.01$ \\
\hline present & $10-25$ & $0.27 \pm 0.01$ & $\mathbf{0 . 5 9} \pm \mathbf{0 . 0 1}$ & $0.55 \pm 0.02$ \\
present & $50-75$ & $0.51 \pm 0.01$ & $\mathbf{0 . 8 1} \pm \mathbf{0 . 0 1}$ & $0.79 \pm 0.01$ \\
present & $100-250$ & $0.69 \pm 0.01$ & $\mathbf{0 . 9 3} \pm \mathbf{0 . 0 1}$ & $0.92 \pm 0.01$ \\
present & $500-750$ & $0.93 \pm<0.01$ & $0.97 \pm<0.01$ & $\mathbf{0 . 9 8} \pm<\mathbf{0 . 0 1}$
\end{tabular}


Table 6: Mean localization error $(\mathrm{m})$ of detections made within the $10 \mathrm{~m}$ radius of the source at $0.1 \%$ FPR using weighted combining. Confidence reflects $95 \%$ intervals. Source intensity $(\mu \mathrm{Ci})$ is evenly distributed within the indicated ranges. The smallest error in each row is represented in bold.

\begin{tabular}{cc|ccc} 
Aperture & Intensity & CEW $(\mathrm{WC})$ & $\mathrm{PIDCEW}(\mathrm{WC})$ & $\mathrm{PID}(\mathrm{WC})$ \\
absent & $10-25$ & $\mathbf{3 . 4 8} \pm \mathbf{0 . 1 2}$ & $3.50 \pm 0.11$ & $5.13 \pm 0.17$ \\
absent & $50-75$ & $2.94 \pm 0.09$ & $\mathbf{2 . 4 4} \pm \mathbf{0 . 0 7}$ & $4.86 \pm 0.16$ \\
absent & $100-250$ & $\mathbf{2 . 3 9} \pm \mathbf{0 . 0 7}$ & $2.44 \pm 0.07$ & $4.69 \pm 0.15$ \\
absent & $500-750$ & $\mathbf{1 . 8 8} \pm \mathbf{0 . 0 5}$ & $1.97 \pm 0.05$ & $4.85 \pm 0.14$ \\
\hline present & $10-25$ & $\mathbf{3 . 8 8} \pm \mathbf{0 . 1 4}$ & $4.30 \pm 0.10$ & $4.92 \pm 0.11$ \\
present & $50-75$ & $3.23 \pm 0.10$ & $\mathbf{3 . 0 6} \pm \mathbf{0 . 0 8}$ & $3.51 \pm 0.09$ \\
present & $100-250$ & $2.78 \pm 0.08$ & $\mathbf{2 . 2 4} \pm \mathbf{0 . 0 6}$ & $2.65 \pm 0.07$ \\
present & $500-750$ & $2.26 \pm 0.06$ & $\mathbf{1 . 7 3} \pm \mathbf{0 . 0 4}$ & $2.15 \pm 0.06$
\end{tabular}

Table 7: Proportion of detections made within the $10 \mathrm{~m}$ radius of the source at $0.1 \%$ FPR using weighted combining. Confidence reflects $95 \%$ intervals. Source intensity $(\mu \mathrm{Ci})$ is evenly distributed within the indicated ranges. The largest proportion in each row is represented in bold.

\begin{tabular}{cc|ccc} 
Aperture & Intensity & $\mathrm{CEW}(\mathrm{WC})$ & $\mathrm{PIDCEW}(\mathrm{WC})$ & $\mathrm{PID}(\mathrm{WC})$ \\
absent & $10-25$ & $\mathbf{0 . 4 3} \pm \mathbf{0 . 0 1}$ & $0.42 \pm 0.01$ & $0.22 \pm 0.01$ \\
absent & $50-75$ & $0.67 \pm 0.01$ & $\mathbf{0 . 8 2} \pm \mathbf{0 . 0 1}$ & $0.25 \pm 0.01$ \\
absent & $100-250$ & $\mathbf{0 . 8 2} \pm \mathbf{0 . 0 1}$ & $\mathbf{0 . 8 2} \pm \mathbf{0 . 0 1}$ & $0.27 \pm 0.01$ \\
absent & $500-750$ & $\mathbf{0 . 9 4} \pm \mathbf{0 . 0 1}$ & $0.93 \pm 0.01$ & $0.27 \pm 0.01$ \\
\hline present & $10-25$ & $0.33 \pm 0.01$ & $\mathbf{0 . 6 3} \pm \mathbf{0 . 0 1}$ & $0.58 \pm 0.01$ \\
present & $50-75$ & $0.54 \pm 0.01$ & $\mathbf{0 . 8 9} \pm \mathbf{0 . 0 1}$ & $0.74 \pm 0.01$ \\
present & $100-250$ & $0.71 \pm 0.01$ & $\mathbf{0 . 9 0} \pm \mathbf{0 . 0 1}$ & $0.86 \pm 0.01$ \\
present & $500-750$ & $0.89 \pm 0.01$ & $\mathbf{0 . 9 7} \pm<\mathbf{0 . 0 1}$ & $0.93 \pm 0.01$
\end{tabular}


ably faithful to the natural physics of such a scenario. We used real world data to ground the parameters and produce a plausible experimental environment within which the considered methods can be compared. We analyzed the performance of spectral (CEW), spatial (PID), and hybrid (PIDCEW) detection algorithms with a Bayesian (BA) and analytic weighted projection (WC) data fusion methodologies. Each approach was simulated both with and without the coded aperture in place so that the impact of the coded aperture could be directly quantified.

Unsurprisingly, our results indicate that algorithm choice is important when considering the utility of coded aperture sensing. In particular, naïvely applying CEW to a detector outfitted with a coded aperture results in a decrease in both threat detection and localization performance. The PID algorithm is able to make use of the coded aperture, giving improved localization performance. In particular, PID and PIDCEW are able to effectively localize sources at greater distances than CEW. Importantly, using the hybrid approach PIDCEW much of the detection performance lost due to partial masking of the sensor surface can be recovered while preserving much of the superior localization capabilities of the coded aperture detector array.

The impact of the data aggregation method is somewhat mixed. Overall, Bayesian aggregation yields better detection performance than weighted combining, in particular when using the top performing scoring algorithms for each sensing context (CEW for unmasked and PIDCEW for masked sensors). Using WC without the coded aperture however, PIDCEW detects consistently better than CEW and localizes with similar accuracy. From the perspective of WC, CEW and PIDCEW are very similar: both can be thought of as a linear system of the form of (7). However, PIDCEW considers each detector element individually rather than summing over them. This produces marginally more consistent scores which partially explains difference in performance.

Despite these subtleties however, when considering either aggregation method, the impact of the coded aperture is directionally consistent: detection performance is somewhat lower but localization performance is superior with the mask. Further, these differences are marginal for strong or nearby sources.

The coded aperture can be thought of as trading information in form of photon counts for partial directional information; i.e. photons that are observed are known not to have come from a direction that is obscured by the aperture. Our primary objective was to characterize the consequence of that trade. Conceptually, this can be accomplished by comparing the performance of the theoretically optimal detection algorithms for each configuration. We take a more practical approach and compare a handful of successful algorithms. Our results demonstrate that the detection performance lost due to coded aperture can be substantially albeit not fully recovered by the alternative algorithms we considered. It may be that yet another algorithm we did not consider could offer detection performance comparable or even superior to CEW. Whatever the case, we have demonstrated that the drop in detection performance is only appreciable for weak/distant sources. In addition, we did not consider evaluating the impact of different shielding or coded aperture patterns. Shielding the back 
and or bottom of the detector may be of interest. Additionally, optimizing the coded aperture pattern for the application may boost performance. A rough analysis of the KL divergence suggests that increasing the variance of the exposure experienced by the detector array should increase performance. A proper treatment of optimizing coded aperture patterns is beyond the scope of this paper. We refer the reader to [2] for a discussion of near-field coded aperture design.

Our simulation framework introduced some simplifications, namely imposing a flat landscape and ignoring the impact of environmental factors due to detector element placement within the array. We expect these simplifications to have little impact on the relative performance of the methods considered. The PID model given in (7) explicitly makes use of the knowledge of no detector element placement impact. Modification of this model to take those effects into account is relatively straightforward. One could replace the ones vector with a vector of deviations from the overall array mean background.

Our investigation only considered a single source template. We expect that other source templates would give similar results, though the magnitude of the differences might change. All of the methods considered here are weakly informed in that they require only a source window $\mathcal{W}$. Source template will impact performance in as much as the linear models used in CEW and PIDCEW make use of the assumption that out-of-window observations do not contain an appreciable number of source counts. This assumption may be more accurate for some source templates than others, even when the window that maximizes the SNR is known. We expect PID to be insensitive to source template provided that background levels and SNR are consistent, since PID does not use spectral shape information.

Our simulation framework was tailored specifically to the RadMAP [20] data and MISTI detection vehicle [15]. Huh, et al. [12] have previously demonstrated the feasibility of localizing sources using the MISTI unit. Their in-silico investigation did not consider the presence of background radiation however. Here, we have demonstrated in-silico evidence for detection and localization of sources in the presence of spatially and temporally (spectrally) varying background.

We restricted inference to only those observations for which the mask was facing hypothetical source positions. Thus, our findings ought to be directionally applicable to dual-sided coded-aperture systems [19] as well. Additionally, many of the methods considered require pre-training regression models for background prediction, which may benefit from radiation mapping exercises [4] for areas of operation.

Our results demonstrate that the coded aperture effects a trade-off between detection and localization, detection performance being decreased and localization performance being increased. The utility of the trade-off is application dependent as different scenarios will place varying emphasis on detection and localization. For instance, maritime [13] or long-range [25] applications may benefit from the ability of coded aperture detectors to localize sources at range. Nonetheless, we have also shown that the drop in detection performance characteristic to coded aperture sensors can be to some extent mitigated algorith- 
mically.

For the roadside threat detection application studied here, PIDCEW with coded aperture yields a significant improvement in likelihood to localize well over CEW without the aperture for all but the strongest sources evaluated. In this scenario this improvement may have little impact on operating procedure once a threat has been detected. Likely a large area around the threat would be locked down in such an event. However, we point out that when the threat detection algorithm gives an alert it will first have to be evaluated to determine whether or not it is a false positive before appropriate action can be taken. Increased localization accuracy may reduce the time it takes to dismiss false positives, improving overall operations.

\section{References}

[1] Helle Karina Aage and U Korsbech. Search for lost or orphan radioactive sources based on nai gamma spectrometry. Applied radiation and isotopes, 58(1):103-113, 2003.

[2] Roberto Accorsi. Design of a near-field coded aperture cameras for highresolution medical and industrial gamma-ray imaging. PhD thesis, Massachusetts Institute of Technology, 2001.

[3] Kevin K Anderson, Kenneth D Jarman, Matthew L Mann, David M Pfund, and Robert C Runkle. Discriminating nuclear threats from benign sources in gamma-ray spectra using a spectral comparison ratio method. Journal of Radioanalytical and Nuclear Chemistry, 276(3):713-718, 2008.

[4] Timothy J Aucott, Mark S Bandstra, Victor Negut, Daniel H Chivers, Reynold J Cooper, and Kai Vetter. Routine surveys for gamma-ray background characterization. Nuclear Science, IEEE Transactions on, 60(2):1147-1150, 2013.

[5] Timothy J Aucott, Mark S Bandstra, Victor Negut, Joseph C Curtis, Daniel H Chivers, and Kai Vetter. Effects of background on gamma-ray detection for mobile spectroscopy and imaging systems. Nuclear Science, IEEE Transactions on, 61(2):985-991, 2014.

[6] Timothy J Aucott, Mark S Bandstra, Victor Negut, Joseph C Curtis, Ross E Meyer, Daniel H Chivers, and Kai Vetter. Impact of detector efficiency and energy resolution on gamma-ray background rejection in mobile spectroscopy and imaging systems. Nuclear Instruments and Methods in Physics Research Section A: Accelerators, Spectrometers, Detectors and Associated Equipment, 789:128-133, 2015.

[7] El E Fenimore and TM Cannon. Coded aperture imaging with uniformly redundant arrays. Applied optics, 17(3):337-347, 1978.

[8] RC Geary. The frequency distribution of the quotient of two normal variates. Journal of the Royal Statistical Society, 93(3):442-446, 1930. 
[9] Stephen R Gottesman and EE Fenimore. New family of binary arrays for coded aperture imaging. Applied optics, 28(20):4344-4352, 1989.

[10] Robert J Hodrick and Edward C Prescott. Postwar us business cycles: an empirical investigation. Journal of Money, credit, and Banking, pages $1-16,1997$.

[11] Berthold KP Horn, Richard C Lanza, Jayna T Bell, and Gordon E Kohse. Dynamic reconstruction. 2010.

[12] Sam Huh, Jonathan Maltz, Dan Gunter, Lucian Mihailescu, and Kai Vetter. Real-time radioactive source localization with a moving codedaperture detector system at low count rates. In Nuclear Science Symposium and Medical Imaging Conference (NSS/MIC), 2012 IEEE, pages 666-673. IEEE, 2012.

[13] Anthony L Hutcheson, Bernard F Phlips, Eric A Wulf, Lee J Mitchell, W Neil Johnson, and Byron E Leas. Maritime detection of radiologi$\mathrm{cal} /$ nuclear threats with hybrid imaging system. In Technologies for Homeland Security (HST), 2013 IEEE International Conference on, pages 360363. IEEE, 2013.

[14] IEEE Nuclear Science Symposium. Using Gaussian Rate Priors with Poisson Data Likelihoods for Improved Detection of Sources of Known Types in Cluttered Background Scenes, Seattle, WA, USA, November 2014.

[15] Lee J Mitchell, BF Phlips, W Neil Johnson, Eric A Wulf, Anthony L Hutcheson, CJ Lister, Kelia D Bynum, Byron E Leas, and Gerald Guadagno. Mobile imaging and spectroscopic threat identification (misti): system overview. In Nuclear Science Symposium Conference Record (NSS/MIC), 2009 IEEE, pages 110-118. IEEE, 2009.

[16] Daniel B Neill. Fast subset scan for spatial pattern detection. Journal of the Royal Statistical Society: Series B (Statistical Methodology), 74(2):337360, 2012.

[17] K Nelson and S Labov. Aggregation of mobile radiation data. Lawrence Livermore National Lab Technical Report, 2010.

[18] Karl Nelson and Simon Labov. Aggregation of mobile data,. Lawrence Livermore National Lab Technical Report, 1(2.2):2-3, 2012.

[19] RD Penny, WE Hood, RM Polichar, FH Cardone, LG Chavez, SG Grubbs, BP Huntley, RA Kuharski, RT Shyffer, Lorenzo Fabris, et al. A dual-sided coded-aperture radiation detection system. Nuclear Instruments and Methods in Physics Research Section A: Accelerators, Spectrometers, Detectors and Associated Equipment, 652(1):578-581, 2011. 
[20] Brian Quiter, Lavanya Ramakrishnan, Mark Bandstra, et al. Grdc: a collaborative framework for radiological background and contextual data analysis. Technical report, Lawrence Berkeley National Laboratory (LBNL), Berkeley, CA (United States), 2015.

[21] Y Román-Montoya, M Rueda, and A Arcos. Confidence intervals for quantile estimation using jackknife techniques. Computational Statistics, 23(4):573-585, 2008.

[22] Prateek Tandon, Peter Huggins, Rob Maclachlan, Artur Dubrawski, Karl Nelson, and Simon Labov. Detection of radioactive sources in urban scenes using bayesian aggregation of data from mobile spectrometers. Information Systems, 57:195-206, 2015.

[23] Shawn Tornga. Mobile, hybrid Compton/coded aperture imaging for detection, identification and localization of gamma-ray sources at stand-off distances. PhD thesis, University of New Mexico, 2013.

[24] Kai Vetter, Dan Chivers, and Brian Quiter. Advanced concepts in multidimensional radiation detection and imaging. In Nuclear Threats and Security Challenges, pages 179-192. Springer, 2015.

[25] Klaus P Ziock, William W Craig, Lorenzo Fabris, Richard C Lanza, Shawn Gallagher, Berthold KP Horn, and Norm W Madden. Large area imaging detector for long-range, passive detection of fissile material. Nuclear Science, IEEE Transactions on, 51(5):2238-2244, 2004.

[26] Klaus-Peter Ziock and Karl E Nelson. Maximum detector sizes required for orphan source detection. Nuclear Instruments and Methods in Physics Research Section A: Accelerators, Spectrometers, Detectors and Associated Equipment, 579(1):357-362, 2007.

[27] KP Ziock, JW Collins, L Fabris, S Gallagher, BKP Horn, RC Lanza, and NW Madden. Source-search sensitivity of a large-area, coded-aperture, gamma-ray imager. IEEE transactions on nuclear science, 53(3):16141621, 2006.

[28] KP Ziock and WH Goldstein. The lost source, varying backgrounds and why bigger may not be better. In Unattended Radiation Sensor Systems for Remote Applications, volume 632, pages 60-70. AIP Publishing, 2002. 